\title{
Routing Optimization in Optical Burst Switching Networks
}

\author{
M. Klinkowski ${ }^{1,2}$, M. Pióro ${ }^{3}$, D. Careglio ${ }^{1}$, M. Marciniak ${ }^{2}$, and J. Solé-Pareta ${ }^{1}$ \\ ${ }^{1}$ Universitat Politècnica de Catalunya (UPC), \\ Advanced Broadband Communication Centre - CCABA \\ Jordi Girona 1-3, 08034 Barcelona, Spain \\ ${ }^{2}$ National Institute of Telecommunications, \\ Department of Transmission and Fibre Technology, \\ 1 Szachowa Street, 04-894 Warsaw, Poland \\ ${ }^{3}$ Warsaw University of Technology (WUT), \\ Institute of Telecommunications, \\ 15/19 Nowowiejska Street, 00-665 Warsaw, Poland \\ \{mklinkow@ac.upc.edu,mpp@tele.pw.edu.pl, careglio@ac.upc.edu, \\ mmarcin@itl.waw.pl,pareta@ac.upc.edu\}
}

\begin{abstract}
This paper addresses the problem of routing optimization in optical burst switching (OBS) networks. We use a simplified analytical model of OBS network with an overall burst loss probability as the primary metric of interest. Since the objective function of optimization problem is nonlinear we propose two solutions based on a non-reduced link load (NR-LL) model and a reduced link load (R-LL) model. In order to find partial derivatives of the cost function we apply a calculation considered previously for circuit-switched networks. We derive exact partial derivatives of NR-LL model and we approximate the partial derivatives of R-LL model. Simulation results demonstrate that our solutions effectively reduce the overall burst loss probability over the shortest path routing. Moreover, in many cases, they over-perform an alternative routing.
\end{abstract}

Keywords: optical burst switching, optimization, routing.

\section{Introduction}

Optical burst switching (OBS) [1] is a promising solution for reducing the gap between switching and transmission speeds in future networks. The client packets are aggregated and assembled into optical burst units in the edge nodes of OBS network. A burst control packet is transmitted in a dedicated control channel and delivered with a small offset-time prior to the data burst. In this way the electronic controller of an intermediate node has enough time both to reserve a wavelength in its output link, usually for the duration time of the incoming burst, and to reconfigure dynamically the switching matrix. When the burst transmission is finished in a node the output wavelength is released for other connections. Such a temporary usage of wavelengths allows for higher resource utilization as well as better adaptation to highly variable input traffic in comparison to optical circuit-switching networks. 
OBS architectures with limited buffering capabilities are susceptible to congestion states. The existence of a few highly congested links may seriously aggravate the network throughput (see e.g. [2]). The congestion can be reduced either by an appropriate network dimensioning or by a proper routing in the network. The dimensioning approach fits the node and link capacities according to the matrix of actual traffic load demands and after such optimization it needs only either a simple shortest path algorithm or a similar mechanism (see e.g. [3]). Some parts of such network, however, may encounter the congestion problem if the traffic demands change. On the contrary, the routing approach introduces some operational complexity since it often needs advanced mechanisms with signalling protocols involved. Nevertheless, the advantage is that it facilely adapts to the changes in the traffic demands.

A great part of the research on routing in OBS networks addresses the problem of alternative, also called deflection, routing (e.g. [2], [4]). In such routing scheme the burst is allowed to be deflected dynamically to an alternative routing path in a node if it contents with another burst on the primary routing path. However, a deflection routing can improve the network performance under low traffic loads it may intensify the burst losses under moderate and high loads [5].

Another approach to the routing problem makes use of the optimization theory and a few works can be found in this area [6], [7], [8]. In OBS network a burst loss probability is the primary metric of interest which adequately represents the congestion state of entire network. An approximated form of the overall burst loss probability, which can be found e.g. in [9], has a nonlinear character which may produce some difficulties in formulating an optimization problem. The solutions presented in works [6]-[8] use a linear programming (LP) formulation which either does not consider the overall burst loss probability as a metric of interest or it takes an approximated form of this metric.

The intention of this work is to fill the gap. Namely, we formulate a nonlinear optimization problem for the routing problem in OBS network. Our objective is to distribute the traffic over a set of pre-established routing paths so as to minimize the overall burst loss probability in the network.

The rest of the paper is structured as follows. In Section 2 we present a routing scenario under the study. In Section 3 we provide a loss model of OBS network. In Section 4 we formulate a nonlinear optimization problem and give the partial derivatives of the cost function. In Section 5 we present simulation results that prove the effectiveness of our solutions. In Section 6 we discuss some implementation issues. Finally we conclude the paper in Section 7.

\section{Routing Scenario}

Consider an OBS network such as that illustrated in Fig. 1. There are finitely many links, labelled $k=1,2, \ldots K$, and link $k$ comprises, for simplicity, a fixed number of wavelengths $C$. A subset $p \subset\{1,2, \ldots K\}$ identifies a path; we define a matrix $\left[A_{k p}\right]$ such that $A_{k p}=1$ if link $k$ belongs to path $p$, and $A_{k p}=0$ otherwise. In the network 
there is a set $P$ of paths pre-established between source $(s)$ and destination $(d)$ nodes. A subset $P_{s d} \subset P$ identifies all the paths originated in node $s$ and terminated in node $d$.

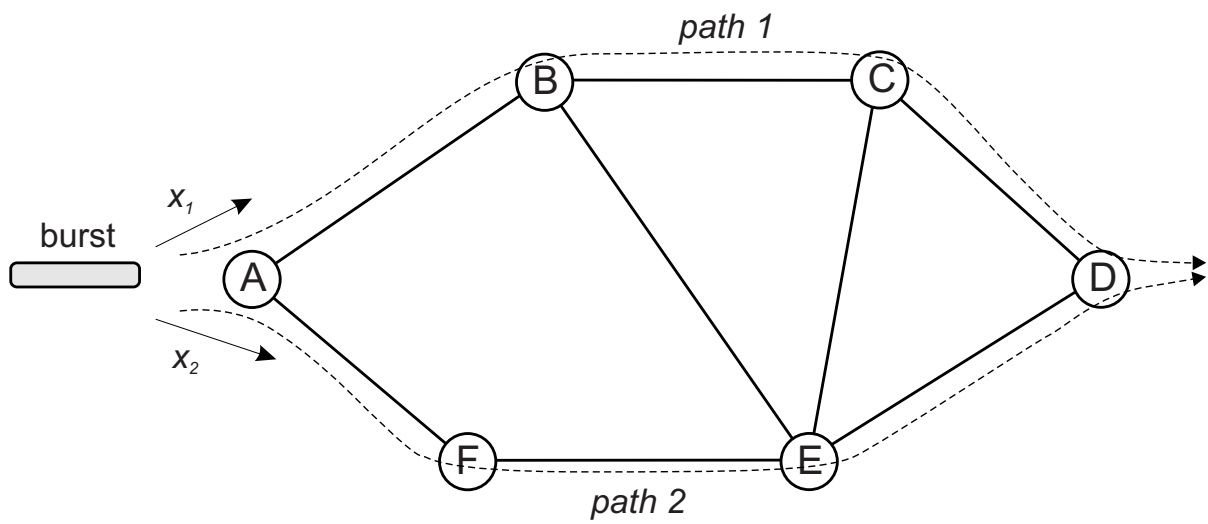

Fig. 1. Example of OBS network with source-based routing; $x_{1}$ and $x_{2}$ are the splitting factors and $x_{1}+x_{2}=1$.

We assume that the network operates with a source-based routing, in that the source node determines the path of a burst that enters the network (see Fig. 1). Moreover, the network uses a multi-path routing where each subset $P_{s d}$ comprises a small number of paths and a burst can take one of those paths. The path selection is performed according to a given splitting factor $x_{p}$, such that the sum of $x_{p}$ of all the paths $p$ belonging to a given subset $P_{s d}$ is equal to 1 .

The traffic pattern is described by the matrix $\left[t_{s d}\right]$ and bursts destined to a given node $d$ arrive to a node $s$ as a Poisson process of (long-term) rate $t_{s d}$. Let $t_{p}=t_{s d}$ for each $p \in P_{s d}$. Thus traffic $v_{p}$ offered to path $p$ can be calculated as:

$$
v_{p}=x_{p} t_{p} .
$$

Here the key factor is the vector $\left(x_{p}\right)$ because it determines the distribution of traffic over the network and it may be selected so that to reduce congestion and to improve overall performance (i.e. in our case the overall burst loss probability). In the next two sections we present analytical derivation of this metric and we formulate an optimization problem.

\section{Loss Model of OBS Network}

A burst going over a path $p$ is blocked and lost if on a given link $k$ that belongs to $p$ there are no free wavelengths. Otherwise a wavelength in the link is reserved for the burst duration and then released immediately after the burst transmission. The reservation holding period is independent of earlier arrival times and holding periods; the holding periods on each link are identically distributed with a mean equal to the mean burst duration, for simplicity we assume that it is equal to 1 . 
Given the difficulty in obtaining an exact formula for the blocking of a burst we assume that each blocking event occurs independently from link to link along any path inside the network (see e.g. [9]).

The independence assumption implies that the offered load to link $k$ is a Poisson process with rate $\rho_{k}$ and so the burst loss probability in link $k$ is:

$$
E_{k}=\operatorname{Erl}\left(\rho_{k}, C\right)=\frac{\rho_{k}{ }^{C}}{C !} \cdot\left[\sum_{i=0}^{C} \frac{\rho_{k}{ }^{i}}{i !}\right]^{-1} .
$$

For a moment let us assume that we have a vector $\left(E_{k}\right)$ given. Thus the approximate loss probability $L_{p}$ of bursts offered to path $p$ satisfies:

$$
L_{p}=1-\prod_{k=1}^{K}\left(1-A_{k p} E_{k}\right),
$$

and the overall burst loss probability $B$ is equal to:

$$
B=\sum_{p \in P} v_{p} L_{p} \cdot\left[\sum_{p \in P} v_{p}\right]^{-1} .
$$

In order to calculate (2) we consider two different models that estimate the traffic $\rho_{k}$ offered to a link, namely a non-reduced link load (NR-LL) model and a reduced link load (R-LL) model.

In the NR-LL model the traffic offered to link $k$ is calculated as a sum of the traffic offered to all the paths that cross this link:

$$
\rho_{k}=\sum_{p \in P} A_{k p} v_{p} .
$$

This formula roughly approximates the volume of traffic offered to a link; nevertheless, it allows easily to find the solution of equation (2).

In the R-LL the traffic offered to link $k$ is obtained as a sum of the traffic offered to all the paths that cross this link diminished by the traffic lost in preceding links along these paths. Thus:

$$
\rho_{k}=\sum_{p \in P} A_{k p} v_{p} \prod_{j=1}^{K}\left(1-S_{p j k} E_{j}\right),
$$

where $S_{p j k}$ equals 1 or 0 depending whether or not link $j$ strictly precedes link $k$ along path $p$, respectively.

In order to solve the formula (6) which incorporates equation (2) we take advantage of the Erlang fixed-point approximation (see e.g. [9]).

\section{Formulation of the Optimization Problem}

From equations (1) and (4) we define a cost function to be the subject of optimization: 


$$
B(x)=\sum_{p \in P} x_{p} t_{p} L_{p},
$$

The optimization problem is formulated as follows:

$$
\min B(x)
$$

subject to:

$$
\begin{aligned}
& \sum_{p \in P_{s d}} x_{p}=1 \quad \forall P_{s d}, \\
& 0 \leq x_{p} \leq 1 \quad \forall p \in P .
\end{aligned}
$$

Since the overall burst loss probability is a nonlinear function of vector $\left(x_{p}\right)$ the cost function is nonlinear as well. According to [10] for solving such optimization problem we can use for instance the modified reduced gradient method described in [11]. In order to achieve an optimal solution it is necessary to compute a direction, which leads to a reduction in the total cost. Such direction is indicated by partial derivatives of the cost function.

\subsection{Calculation of Partial Derivatives in NR-LL model}

The partial derivatives in NR-LL model can be derived directly from (1)-(5). Their computation, however, may be time-consuming in larger networks since $|P|$ partial derivatives have to be recalculated. Instead we use a similar derivation like the one proposed by F. Kelly for circuit-switched (CS) networks [12]. Due to space limitation here we provide only the final form of the solution.

Let for each link $k$ :

$$
c_{k}=\left[E\left(\rho_{k}, C-1\right)-E\left(\rho_{k}, C\right)\right] \cdot \sum_{p: k \in p} x_{p} t_{p}\left(1-L_{p}\right) .
$$

Then

$$
\left.\frac{d}{d x_{i}} B(x)=t_{i}\left[L_{i}+\sum_{k \in i} c_{k}\right)\right] .
$$

\subsection{Calculation of Partial Derivatives in NR-LL model}

The application of Kelly's calculation to NR-LL model in OBS network may be quite complex. Therefore we take the derivation initially obtained for CS network model and use it as a rough approximation. 
Let $c=\left(c_{1}, c_{2}, \ldots, c_{K}\right)$ be the (unique) solution to the equation:

$$
c_{k}=\left[E\left(\rho_{k}, C-1\right)-E\left(\rho_{k}, C\right)\right] \cdot\left(1-B_{k}\right)^{-1} \sum_{p: k \in p} x_{p} t_{p}\left(1-L_{p}\right)\left(1-\sum_{j \in p-\{k\}} c_{j}\right) .
$$

Then

$$
\frac{d}{d x_{i}} B(x) \approx t_{i}\left[1-\left(1-L_{i}\right)\left(1-\sum_{k \in i} c_{k}\right)\right]
$$

The formula (14) corresponds strictly to CS network case. Nevertheless, as the numerical examples we used for this study shows it always yields, in OBS network, to the same near-optimal solution of the optimization problem. Moreover, for small networks, where it is feasible to compute direct partial derivatives, the corresponding values of the optimized cost function are identical.

The conformity of results under NR-LL model is kept as well.

We can also observe that low values of the cost function (7) lead to the same nearoptimal solutions in both NR-LL and R-LL models.

\section{Performance}

We evaluate the performance of our routing scheme in an event-driven simulator. In order to find a splitting vector $\left(x_{p}\right)$ that yields to a near-optimal routing in both NR-LL and R-LL models we use a solver fmincon for constrained nonlinear multivariable functions available in the Matlab environment. Then we apply this vector in the simulator. The optimized NR-LL and R-LL routing is compared with two other routing strategies, namely with a simple shortest path (SP) routing and a by-pass routing (BP) which is an alternative-like routing (see [2] for more details).

\subsection{Evaluation Scenario}

Two network topologies are studied, namely a Simple network topology (see Fig. 3a) and the NSFnet network topology (see Fig. 3b). We assume that the links are bidirectional and each link multiplexes $C=32$ wavelengths if not specified otherwise. Each node acts as an edge and a core node. We consider 2 paths per each sourcedestination pair of nodes. The paths are pre-established according to the Dijkstra algorithm (with regard to the number of hops) and they are not necessarily disjoint. In SP routing only 1 path is available.

The nodes are capable to perform a full wavelength conversion according to the random wavelength-selection algorithm. There is no FDL buffering for the burst contention resolution.

Regarding the traffic modelling, each node is capable to generate a burst destined to any other node with the uniform distribution. Each source node has $C$ input 
wavelengths with the input traffic per a wavelength equal to $\rho$ (this parameter is specified later). The burst inter-arrival times to the network are exponentially distributed. The mean burst duration is $1 \mathrm{~ms}$. We assume that the source nodes do not buffer the bursts after completing their aggregation.

All the simulation results have $99 \%$ level of confidence.

a)

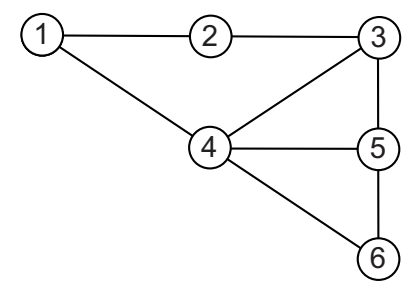

b)

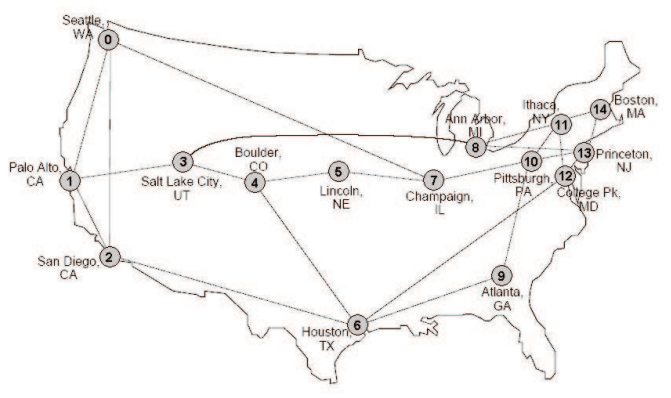

Fig. 2. Network topologies; a) Simple, b) NSFnet.

\subsection{Network Performance}

In Fig. 3a) we can see the overall burst blocking probability in the function of traffic load $(\rho)$ obtained in the Simple network for different routing strategies. The number of input wavelengths as well as the number of wavelengths in a link is equal to 8 . We can observe that both BP and NR-LL and R-LL routing offer a similar performance and all of them outperform SP routing.

Fig. 3b) presents the performance obtained in the Simple network with 32 wavelengths per link. Now the optimized routing outperforms both BP and SP routing, however, the last one is the worst.
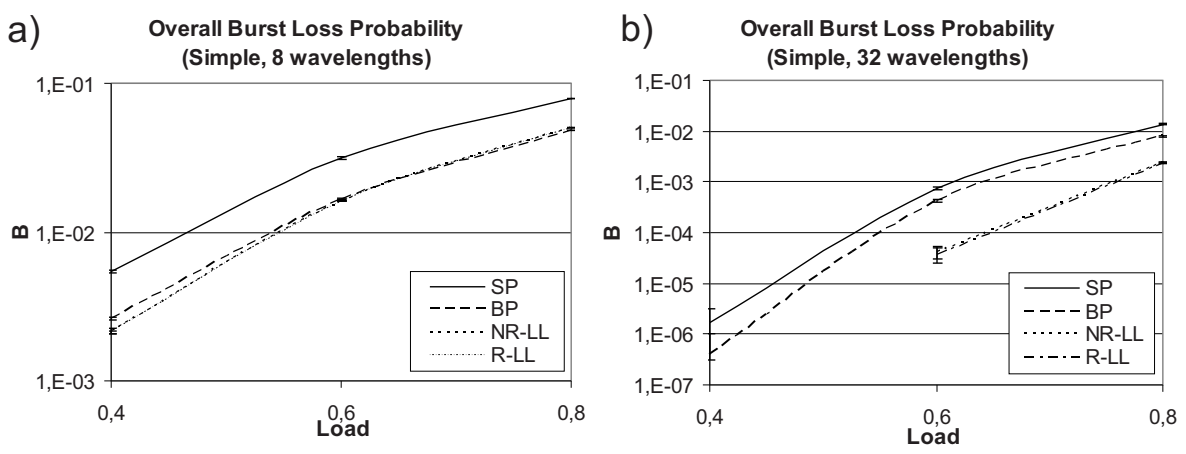

Fig. 3. Comparison of routing schemes in the Simple network; a) $C=8$, b) $C=32$. 
Finally in Fig. 4) we compare the performance in larger NSFnet network. The number of wavelengths is equal to 32. Similarly as in the previous case the lowest burst loss probabilities are achieved with the optimized routing. The BP routing is only a bit better than the SP routing. Such low performance can be explained by a small number of alternative paths (1 path) available in BP routing.

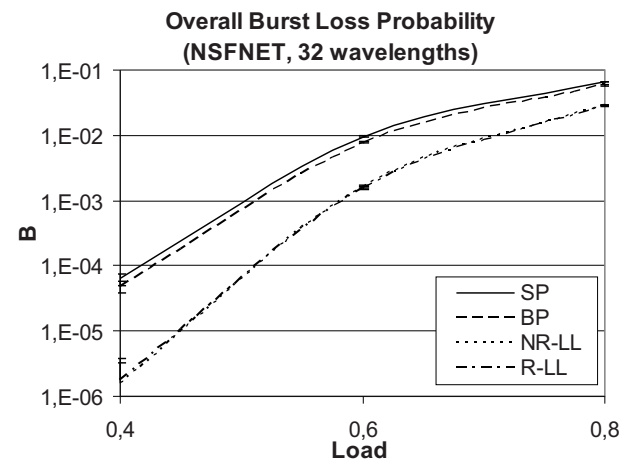

Fig. 4. Comparison of routing schemes in the NSFnet network $(C=32)$.

Note that both NR-LL and R-LL routing offer the same performance without regard to the network and traffic load scenarios. It is because under low link losses (as in the provided examples) the NR-LL model approximates well the traffic offered to a link. As a result both the NR-LL and R-LL models give similar results.

\section{Implementation Issues}

The proposed optimization framework can be used to calculate a traffic splitting vector that determines the distribution of traffic over the network in a multi-path source based routing scenario. We assume that there is a virtual path topology preestablished that comprise, for instance, a limited number of shortest paths between each pair of source-destination nodes. Such virtual topology can be built in a labelled OBS (LOBS) architecture [13].

Routing optimization can be executed either in centralized or in decentralized way.

Centralized optimization can be used in a static (pre-planed) routing, where the traffic distribution is calculated based on a given (long-term) matrix of demands. Then either a periodic or a threshold-triggered update of the splitting vector can be performed if the matrix of demands changes. Both NR-LL and R-LL optimization models can be applied for this purpose.

A distributed routing should react rapidly to a local disturbance at the point of the disturbance, with slower adjustments in the rest of the network. Similarly like it was proposed for circuit-switched networks [12] the formulas (13) and (14) can be used to design a distributed adaptive routing algorithm for OBS network.

In such distributed adaptive routing we assume that in the network there is the possibility of limited communication between the nodes. The nodes are capable to 
measure the loads carried through the links and the source nodes are able to measure the loads carried on the paths. Moreover, there is a (limited) arithmetical processing ability for each link and route, which may be distributed over the nodes of the network; for example the processing for routes might be carried out at the sources nodes. Then the measurements of actual loads together with computing (13) and (14) can be used to implement a decentralized hill-climbing search procedure able gradually to vary routing patterns in response to changes in the demands placed on the network (see [12] for details).

\section{Conclusions}

In this paper the problem of routing optimization in optical burst switched networks with multi-path source-based routing is studied. In this context two different network models are defined, namely with non-reduced link load calculation (NR-LL) and with reduced link load (R-LL) calculation. For both of them a nonlinear optimization problem is formulated and solved.

Simulation results demonstrate that both models equally well find a solution for our routing problem. As a result the traffic is effectively distributed over the network and the network-wide burst loss probability is reduced. The optimized routing always performs better than the shortest path routing. Moreover, it outperforms an alternative routing in a network with high number of wavelengths per link.

Both proposed models can be applied to optimize the distribution of traffic in a centralized way, e.g. in a static routing. Moreover, R-LL model can be used in adaptive distributed routing as it was presented in Kelly's work.

For the purpose of optimization problem we provide the exact partial derivatives in NR-LL model. Moreover, we approximate them in R-LL model by the partial derivatives corresponding to the circuit-switched network model. All the numerical examples we used in this work show that these derivatives lead to a near-optimal solution in OBS network. Nevertheless, the study will be continued in order to prove the correctness of approximation used in R-LL model for any OBS network scenario. The accuracy of OBS network loss models and the properties of the cost function (concavity/convexity or neither of them) will be investigated as well.

The issue not addressed in this paper and related with the multi-path source routing is the problem of out-of-order burst arrival. The burst reordering is common for any multi-path or deflection routing scenario and dedicated mechanisms have to be introduced in order to cope with this problem.

Acknowledgments. The results presented in this work were obtained during a joint Short Term Scientific Mission of the actions COST 293 ("Graphs and algorithms in communication networks") and COST 291 ("Towards digital optical networks"). This work has been partially funded by the MEC (Spanish Ministry of Education and Science) under the CATARO project (Ref. TEC2005-08051-C03-01). 


\section{References}

1. Qiao, C., Yoo, M.: Optical Burst Switching (OBS) - a New Paradigm for an Optical Internet. Journal of High Speed Networks, Vol. 8. No. 1. (1999) 69-84

2. Klinkowski, M., Herrero, F., Careglio, D., Solé-Pareta, J.: Adaptive Routing Algorithms for Optical Packet Switching Networks. Proceedings of 9th IFIP Working Conference on Optical Network Design and Modelling ONDM2005 (2005)

3. Köhn, M., Gauger, C.M.: Dimensioning of SDH/WDM Multilayer Networks. Beiträge zur 4. ITG-Fachtagung Photonische Netze. (2003) 29-33

4. Cameron, C., Zalesky, A., Zukerman M. : Shortest Path Prioritized Random Deflection Routing (SP-PRDR) in Optical Burst Switched Networks, ICST International Workshop on Optical Burst Switching (WOBS). San Jose (2004)

5. Zalesky, A. Vu, H. L., Rosberg, Z., Wong, E. M., Zukerman, M.: Reduced Load Erlang Fixed Point Analysis of Optical Burst Switched Networks with Deflection Routing and Wavelength Reservation. Proceedings of the First International Workshop on Optical Burst Switching (2003)

6. Zhang, J. et al.: Explicit Routing for Traffic Engineering in Labelled Optical Burst-Switched WDM Networks. International Conference on Computational Science ICCS (2004)

7. Teng, J., Rouskas, G.: Traffic Engineering Approach to Path Selection in Optical Burst Switching Networks. Journal of Optical Networking, Vol. 4. No. 11. (2005)

8. Hyytia, E., Nieminen, L.: Linear Program Formulation for Routing Problem in OBS Networks. Proceedings of the 9th IEEE Symposium on Computers and Communications (ISCC 2004)

9. Zukerman, M. et al: Blocking Probabilities of Optical Burst Switching Networks Based on Reduced Load Fixed Point Approximations. Proceedings of INFOCOM Conference (2003)

10.Pioro, M., Wallstrom, B.: Multihour Optimization of Non-Hierarchical Circuit Switched Communication Networks with Sequential Routing. 11th International Teletraffic Congress ITC-11 (1985)

11.Harris, R.J.: The Modified Reduced Gradient Method for Optimally Dimensioning Telephone Networks. Australian Telecom. Research. Vol. 10. No. 1. (1976) 30-35

12.Kelly, F. P.: Routing in Circuit-Switched Networks: Optimization, Shadow Prices and Decentralization. Advanced Applied Probability, Vol. 20. (1988) 112-144

13.Qiao, C.: Labeled Optical Burst Switching for IP-over-WDM Integration. IEEE Communication Magazine. December (2000) 